\title{
On the importance of hydrodynamic interactions in polyelectrolyte electrophoresis
}

\author{
K Grass* $\quad$ C Holm ${ }^{*}, \dagger$
}

September 14, 2021

\begin{abstract}
The effect of hydrodynamic interactions on the free-solution electrophoresis of polyelectrolytes is investigated with coarse-grained molecular dynamics simulations. By comparing the results to simulations with switched-off hydrodynamic interactions, we demonstrate their importance in modelling the experimentally observed behaviour. In order to quantify the hydrodynamic interactions between the polyelectrolyte and the solution, we present a novel way to estimate its effective charge. We obtain an effective friction that is different from the hydrodynamic friction obtained from diffusion measurements. This effective friction is used to explain the constant electrophoretic mobility for longer chains. To further emphasize the importance of hydrodynamic interactions, we apply the model to end-labeled free-solution electrophoresis.
\end{abstract}

PACS numbers: 82.35.Lr, 47.57.Ng, 82.35.Rs, 82.56.Lz, 82.56.Jn, 87.15.ap

Submitted to: J. Phys.: Condens. Matter

\section{Introduction}

Nowadays, electrophoretic separation methods are widely applied to study polyelectrolytes (PEs) such as proteins, DNA and synthetic polymers [1, 2, 3]. While there exist several theories [4, 5, 6, 7] that have been successfully used to describe qualitatively the experimentally observed behaviour of various PEs, there are still many open problems to address.

\footnotetext{
*Frankfurt Institute for Advanced Studies, Goethe University, Ruth-Moufang-Strasse 1, D-60438 Frankfurt am Main, Germany, grass@fias.uni-frankfurt.de

${ }^{\dagger}$ Max-Planck Institute for Polymer Research, Ackermannweg 10, D-55128 Mainz, Germany
} 
Recent experiments on strongly charged flexible PEs, such as polystyrene sulfonate (PSS) and single-stranded DNA (ssDNA) of well defined length have shown a characteristic behaviour for the short chain free-solution mobility $\mu[8,9,10,11]$ : after an initial increase of the mobility with increasing length, $\mu$ passes through a maximum, and then decreases towards a constant mobility for long chains.

The increase for short chains and the long-chain limit can be explained within the theoretical approaches, but the origins of the maximum for intermediate chains remain indistinct. To some extent this can be attributed to the simplifying assumptions made in those models regarding the interplay of the various interactions: the Coulomb interaction between the charged PE monomers and its counterions, the external electric field likewise acting on the charges, and the hydrodynamic interactions with the solvent.

To provide a fundamental understanding of the involved dynamics, we believe it is mandatory to study the effects of these forces on a microscopic level, thereby taking into account full electrostatic as well as hydrodynamic interactions. Continuing the work of [12], we use coarse-grained molecular dynamics simulations to determine the transport coefficients and structural properties of strong polyelectrolytes in free-solution electrophoresis. We characterize the hydrodynamic interactions between solvent and solute on a microscopic level, and determine the relevant length scale for these interactions.

The article is structured as follows: in section 2 we introduce the simulation model and specify all relevant parameters. After comparing the simulation results to experimental measurements of the PSS transport coefficients for varying length (Section 3.1), we switch off hydrodynamic interactions to characterize the behaviour in absence of them. In section 3.4 we introduce the concept for an effective friction of the polyelectrolyte counterion compound with the surrounding solvent. To quantify the effective friction, we propose a novel way to estimate the effective charge. Finally, we show that the effective friction, measured in this way, deviates from the hydrodynamic friction that can be obtained from diffusion measurements, and that this deviation is the reason for the observable length independent mobility of long polyelectrolyte chains. Finally, in section 3.7, we demonstrate, how increasing the effective friction by a drag label can restore size dependent mobility. We finish this paper with concluding remarks.

\section{Simulation model}

We use a charged bead-spring model within the Espresso package [13], to study the behaviour of flexible linear PEs of different lengths. All parameters are given in reduced units with energy scale $k T=1.0$ and relevant length scale $\sigma_{0}$ that is used to match the model to a specific polyelectrolyte. For this paper, we chose $\sigma_{0}=2.5 \AA$, which is the distance between two charges along a fully sulfonated PSS backbone. The simulation time step is $\tau_{0}=0.01$.

The PE is comprised of $N$ negatively charged monomers carrying a charge of $-1 e_{0}$, where $e_{0}$ is the elementary charge.

The monomers are connected by finitely extensible nonlinear elastic (FENE) bonds $U_{\mathrm{FENE}}=$ $\frac{1}{2} k R^{2} \ln \left(1-\left(\frac{r}{R}\right)^{2}\right)$, with stiffness $k=30$, and maximum extension $R=1.5$, and with $r$ being the distance between the interacting monomers [14]. Additionally, a truncated Lennard- 
Jones or Weeks-Chandler-Anderson (WCA) potential $U_{\mathrm{LJ}}=4 \epsilon\left(\left(\frac{\sigma}{r}\right)^{1} 2-\left(\frac{\sigma}{r}\right)^{6}\right)$, with depth $\epsilon=0.25$ and width $\sigma=1$, is used for excluded volume interactions [15].

For charge neutrality, $N$ monovalent counterions of charge $+1 e_{0}$ are added that are subject to the same LJ potential. All particles have the same size.

The simulations are carried out under periodic boundary conditions in a rectangular simulation box. The size $L$ of the box is varied to achieve a constant monomer concentration $c_{\mathrm{PE}}$ and counterion concentration $c_{\mathrm{CI}}$ independent of chain length. To match experimental conditions with a PSS monomer concentration of $1 \mathrm{~g} / 1$ or $5 \mathrm{mM}$, we vary $L$ between $34(N=2)$ and 89 $(N=32)$.

Full electrostatic interactions are calculated with the particle-particle-particle mesh (P3M) algorithm [16]. The Bjerrum length $l_{B}=e_{0}^{2} / 4 \pi \epsilon k T=2.84$ in simulation units corresponds to $7.1 \AA$ (the value for water at room temperature).

To account for hydrodynamic interactions (HI), we frictionally couple all particles to a lattice Boltzmann fluid as detailed in [17]. The modeled fluid has a kinematic viscosity $\nu=$ 3.0 , a fluid density $\rho=0.864$, and is discretized by a grid with spacing $a=1.0$. The coupling parameter is $\Gamma_{\text {bare }}=20.0$.

In order to further characterize the impact of HI on the system's dynamics, we compare to simulations with a simple Langevin thermostat that does not recover long-range hydrodynamic interactions between the monomers, but only offers local interactions with the solvent. We set the friction parameter $\Gamma_{0}=15.34$ to match the single particle mobility of the Langevin system to the one with full $\mathrm{HI}$.

\subsection{Determining transport coefficients}

Using this model, we determine the single chain diffusion coefficient $D$ and the electrophoretic mobility $\mu$ of the model PE from simulations without an applied external field using the following Green-Kubo relations:

$$
\begin{gathered}
D=\frac{1}{3} \int_{0}^{\infty}\left\langle\overrightarrow{v_{c}}(\tau) \cdot \overrightarrow{v_{c}}(0)\right\rangle d \tau \\
\mu=\frac{1}{3 k_{B} T} \sum_{i} q_{i} \int_{0}^{\infty}\left\langle\overrightarrow{v_{c}}(\tau) \cdot \overrightarrow{v_{i}}(0)\right\rangle d \tau
\end{gathered}
$$

Here, $\overrightarrow{v_{c}}$ is the center of mass velocity of the PE, $\overrightarrow{v_{i}}$ is the velocity of every charged particle in the system, i.e. monomers of the PE and associated counterions, and $q_{i}$ is the corresponding charge. The ensemble average $(\langle\ldots\rangle)$ is taken over $10^{4}$ statistically independent samples. The integrations use analytic fits for the slowly converging long-time tails.

The advantage of using a Green-Kubo relation (2) for the electrophoretic mobility is that we can obtain both transport coefficients from the same simulation trajectories at zero field. Doing so we avoid conformational changes to the chain structures or the counterion distributions by an artificially high external electric field, which is sometimes used in other simulations to separate the directed electrophoretic motion from the Brownian motion within reasonable 
computing time. This approach was successfully applied in simulations to determine the electrophoretic mobility of charged colloids [18, 19].

\section{Results}

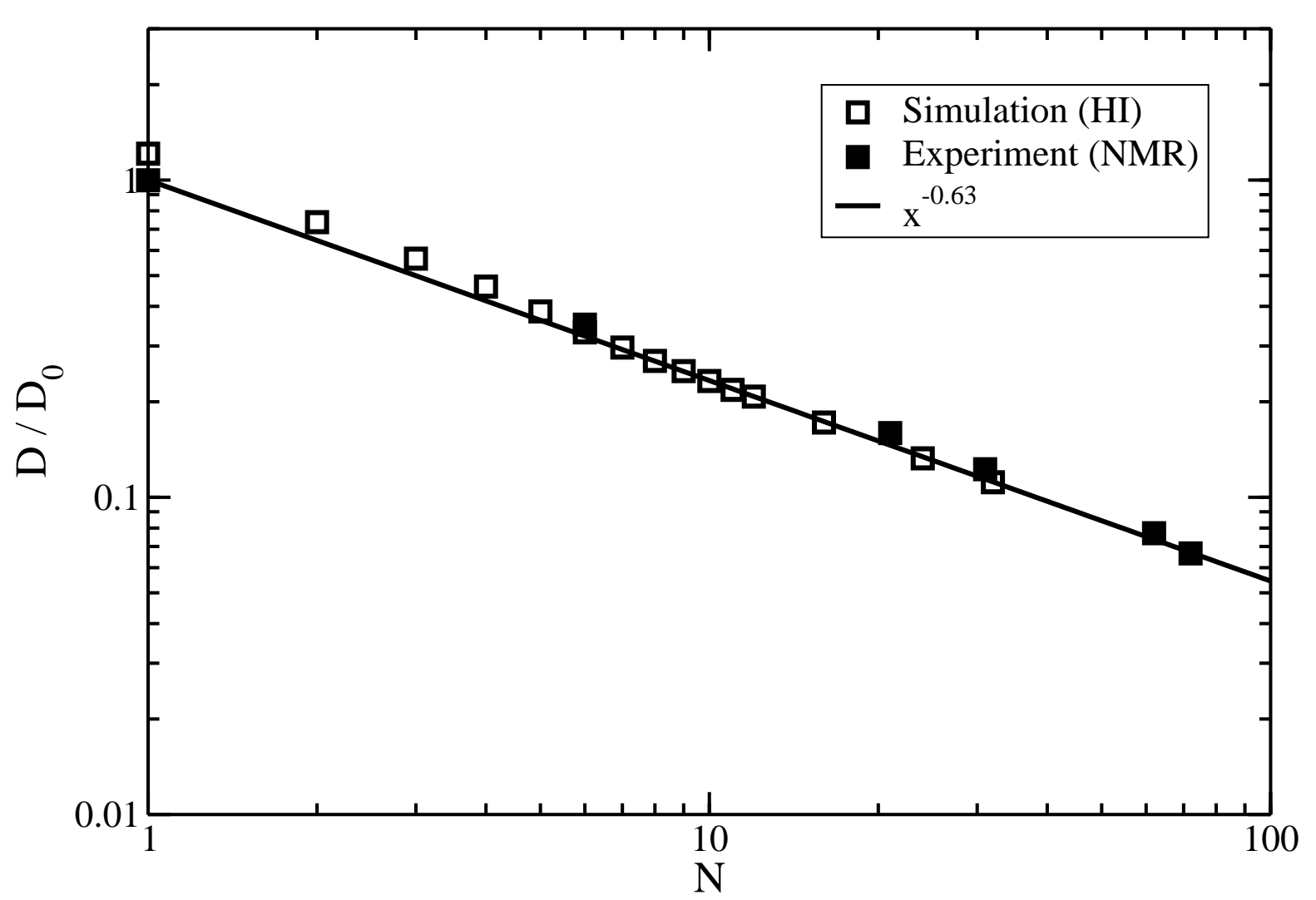

Figure 1: The normalized diffusion coefficient $D / D_{0}$ for PSS of different lengths $\mathrm{N}$ as obtained by electrophoresis NMR agrees with the simulation results with full hydrodynamic interactions $(\mathrm{HI})$.

In an earlier publication this year [12], we compared the simulation results to experimental data for short PSS obtained by two different experimental methods, namely, capillary electrophoresis [8, 20] and pulsed field gradient or electrophoretic NMR [21, 22, 23, 24] 1 .

In Figure 1, we compare the diffusion coefficient $D$ determined by simulations to the experimental results. Both results are in good agreement and exhibit a power law scaling $D=D_{0} N^{m}$, with an scaling exponent $m=0.63 \pm 0.01$, which agrees with previous results

${ }^{1}$ Please refer to [12] for details on the experimental conditions. 


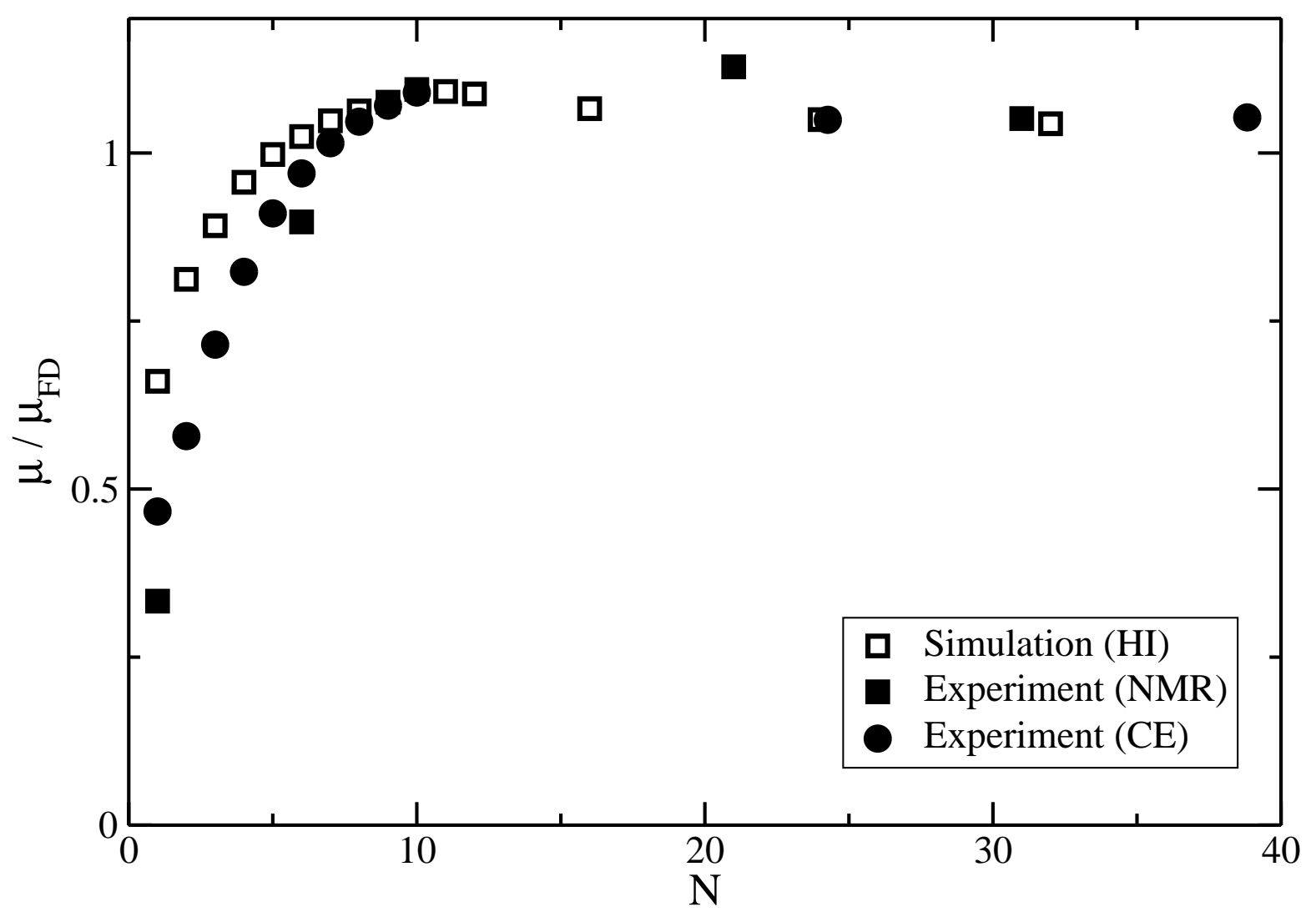

Figure 2: Including hydrodynamic interactions (HI), the normalized electrophoretic mobility $\mu / \mu_{\mathrm{FD}}$ as a function of the number of repeat units $N$ obtained in simulations reproduce a maximum for intermediate chains as well as the long-chain behaviour observed in experiments. 
[11, 25, 26, 27]. Here, the simulated data is normalized by $D_{0}=0.052$, the monomer diffusion as obtained by a power law fit, and the experimental data by the monomer diffusion coefficient of $D_{0}=5.7 \cdot 10^{-10} \mathrm{~m}^{2} / \mathrm{s}$.

Figure 2 shows the electrophoretic mobility $\mu$ normalized by $\mu_{\mathrm{FD}}$, the constant (not lengthdependend) value for long chains. We compare the simulation results to two diffent experimental data sets. The simulation results reproduce a maximum for intermediate chains as well as the long-chain behaviour (the constant mobility for long chains) observed in experiments, if the hydrodynamic interactions are properly accounted for.

\section{Sheanno nf hydrndynamir intarantinne}

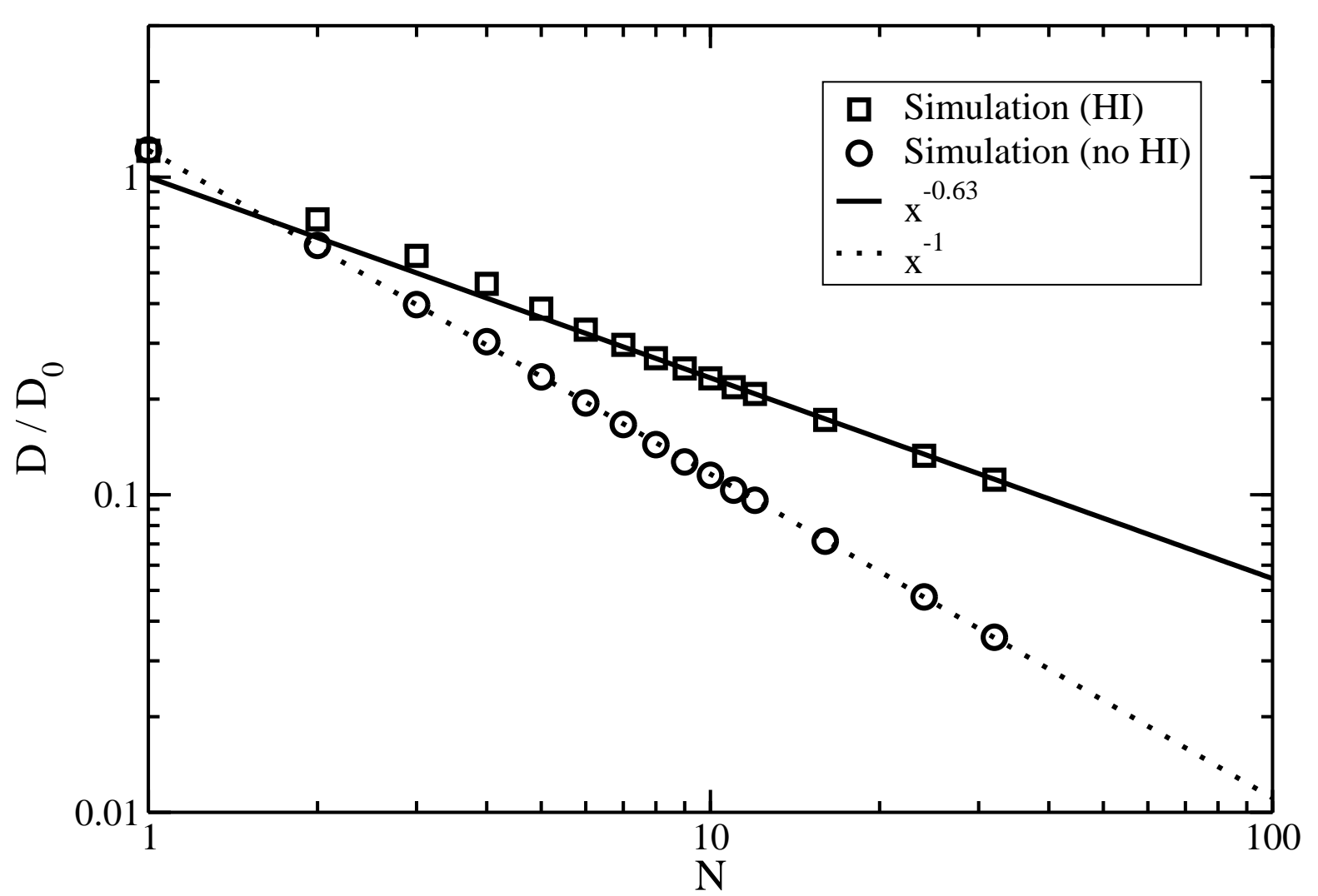

Figure 3: The normalized diffusion coefficient $D / D_{0}$ with and w ithout hydrodynamic interactions (HI). With $\mathrm{HI}$ the experimentally observed sca ling is recovered; without $\mathrm{HI}$ the chain diffusion is Rousse-like.

Figure 3 illustrates the difference in the scaling of the diffusion coefficient with and without hydrodynamic interactions. For the latter, Rousse behaviour is observed with a scaling exponent of $m=1.02 \pm 0.02$ for the chain diffusion. Likewise, as shown in figure 4, the neglect of 


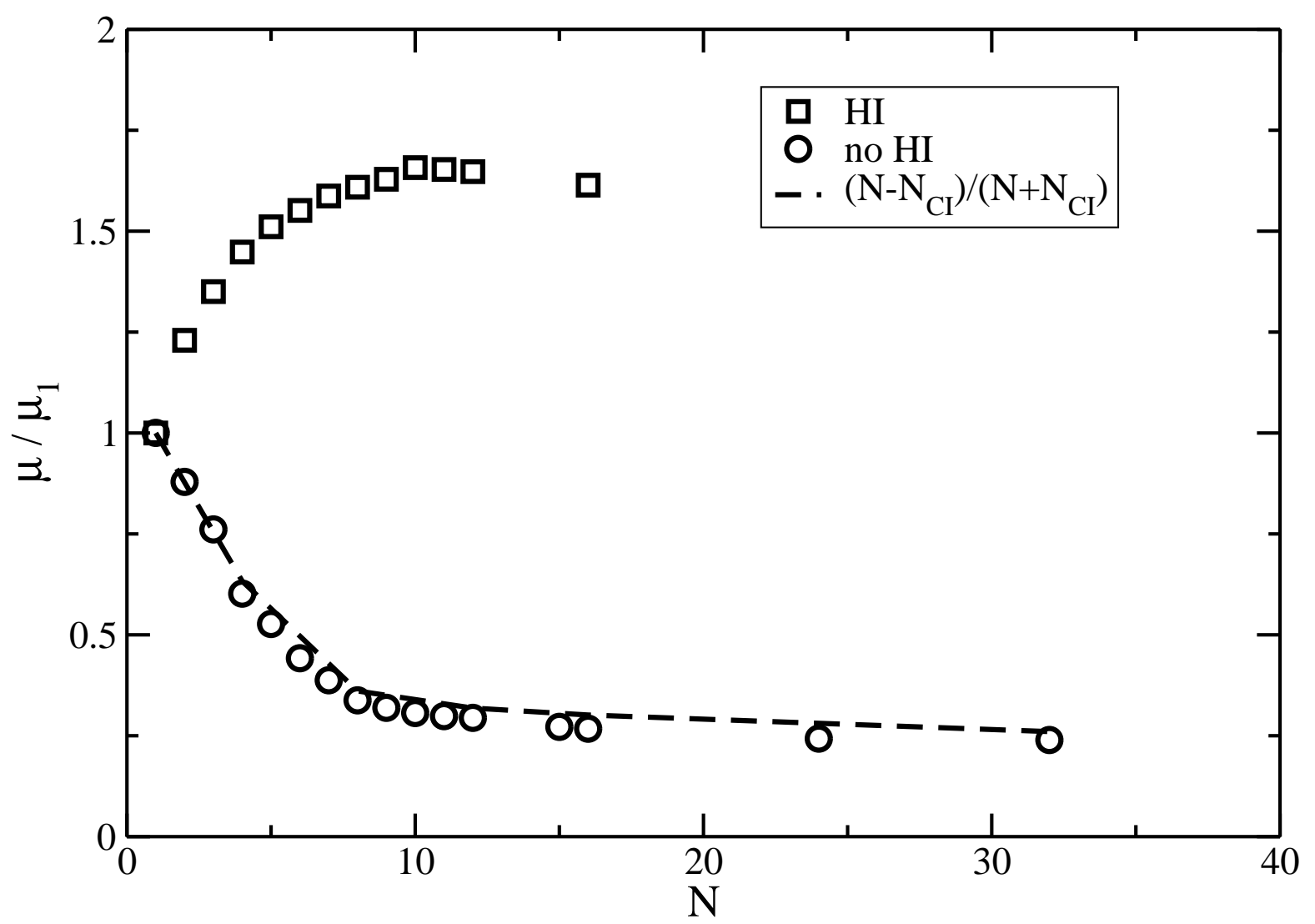

Figure 4: The normalized electrophoretic mobility without hydrodynamic interactions (HI) strongly deviates from the one obtained with HI. The plotted curve, equation (5), describes this observation by using the local force picture. 
hydrodynamic interactions leads to a decreasing electrophoretic mobility for short chains and a constant value for long chains. The same observations were made in a recent publication by Frank and Winkler [28].

\subsection{Local force balance}

The behaviour in absence of hydrodynamic interactions can be understood in a local force balance picture. A strongly-charged polyelectrolyte such as PSS is surrounded by a cloud of oppositely charged counterions, some of which move with the chain, thus forming a molecular compound with the PE that has a reduced electric charge. This effect is also know as counterion condensation [29]. Without hydrodynamic interactions, every particle of this compound is subject to the same frictional force specified by the Langevin thermostat. For a not to strong applied electrical field $E$, the electrophoretic mobility can be obtained by $\mu=v / E$, where $v$ is the steady-state velocity of the compound (PE and co-moving counterions). The two acting forces, the solvent drag force $F_{\text {Solvent }}$ and the electric driving force $F_{\text {Field }}$, are of equal magnitude and opposite direction. We now define $N_{\mathrm{CI}}$ to be the number of co-moving counterions. This leads to the following equation for the forces, where $\pm Q$ is the charge of the monomers and the counterions, respectively.

$$
\begin{aligned}
F_{\text {Solvent }} & =-\Gamma_{0} v\left(N+N_{\mathrm{CI}}\right) \\
F_{\text {Field }} & =Q E\left(N-N_{\mathrm{CI}}\right)
\end{aligned}
$$

From this we can obtain the following expression for the electrophoretic mobility in absence of hydrodynamic interactions, where $\mu_{0}=1 / \Gamma_{0}$ is the mobility of a single monomer:

$$
\frac{\mu}{\mu_{0}}=\frac{N-N_{\mathrm{CI}}}{N+N_{\mathrm{CI}}}
$$

For plotting (5) in figure 4, we obtain $N_{\mathrm{CI}}$ by counting the average number of counter-ions found within $2 \sigma_{0}$ of the chain. The local force picture successfully describes the observed behaviour in absence of hydrodynamic interactions.

In the linear response regime neither the chain structure nor the counterion distribution is affected by the presence of hydrodynamic interactions. Thus, the differences displayed in figures 3 and 4 can only be attributed to different frictional forces with the solvent acting on the PE-counterion compound. In the remainder of this paper, we will investigate this in more detail.

\subsection{Introducing hydrodynamic friction}

Figure 4 shows that the mobility strongly increases when hydrodynamic interactions are taken into account. In other words, the frictional forces the compound experiences from the solvent are reduced by the hydrodynamic interactions between the monomers and the counterions. The solvent interactions are no longer local, thus (3) and (4) have to be modified:

$$
F_{\text {Solvent }}=-\Gamma_{\text {eff }}\left(N, N_{\mathrm{CI}}\right) v
$$




$$
F_{\text {Field }}=Q_{\text {eff }}\left(N, N_{\mathrm{CI}}\right) E
$$

Here, $\Gamma_{\text {eff }}$ and $Q_{\text {eff }}$ are the a priori unknown effective friction and effective charge of the compound, that depend on the degree of polymerization $N$ of the PE chain as well as on the number of co-moving counterions $N_{\mathrm{CI}}$. Combining both equations leads to a general expression for the electrophoretic mobility:

$$
\mu=\frac{Q_{\text {eff }}\left(N, N_{\mathrm{CI}}\right)}{\Gamma_{\mathrm{eff}}\left(N, N_{\mathrm{CI}}\right)}
$$

Since the general functional dependence of $\Gamma_{\text {eff }}$ on $N$ and $N_{\mathrm{CI}}$ in the presence of hydrodynamic interactions is non trivial, in all but limiting cases, (8) cannot be used to determine the electrophoretic mobility analytically. However, it can be applied to determine the effective friction from the electrophoretic mobility and the effective charge:

$$
\Gamma_{\text {eff }}=Q_{\text {eff }} / \mu .
$$

\subsection{Estimating the effective charge}

As before, the electrophoretic mobility is determined by (2), leaving the task to estimate the effective charge from the simulation. There are several possible ways to determine the number of counterions that shield the bare charge of the polyelectrolyte. In section 3.3, we already used the straightforward way of counting the number of counterions within a tube of radius $2 \sigma$ around the chain.

$$
Q_{\text {eff }}^{(1)}=N-N_{\mathrm{CI}}(d<2 \sigma)
$$

This method has the draw back, that the threshold, i.e. the size of the tube, is arbitrarily defined. Another frequently used way is the inflection criterion [30, 31], in which the integrated radial counterion distribution is calculated and the inflection point used as a threshold.

Alternatively, we can rewrite (5) and use the electrophoretic mobility without hydrodynamic interactions to calculate the effective charge.

$$
Q_{\mathrm{eff}}^{(2)}=N\left(1-\frac{1-\mu \Gamma_{0}}{1+\mu \Gamma_{0}}\right)
$$

This definition of the effective charge does not have any free parameters and since simulations without hydrodynamic interactions are computationally inexpensive, we can determine $Q_{\text {eff }}$ with high accuracy in this way. Further analysis showed that $Q_{\text {eff }}$ is insensitive to hydrodynamic interactions in the linear response regime.

In figure 5], we compare both estimators for the effective charge. For short chains, both estimators agree and coincide with the bare, unscreened charge of the polyelectrolyte. In this regime, no counterion condensation is observed.

For intermediate chains, the effective charge is reduced as it deviates from the bare charge and tends towards the Manning prediction. Here, the condensation parameter for the PSS system is $\xi=3.12$. In this regime, the simple estimator $Q_{\mathrm{eff}}^{(1)}$ measures a higher effective 


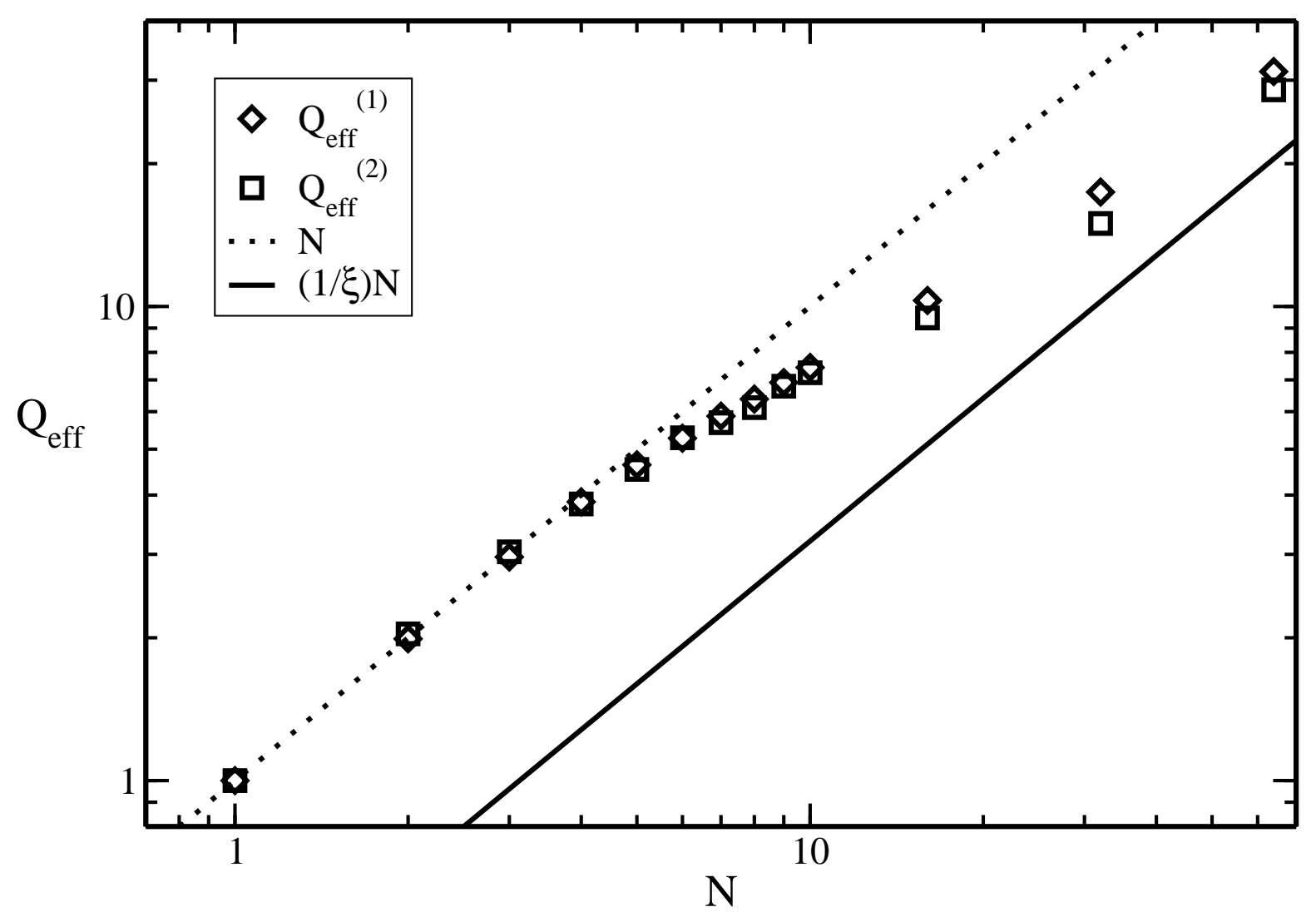

Figure 5: The effective charge $Q_{\text {eff }}$ as a function of chain length for both estimators $Q_{\mathrm{eff}}^{(1)}$ and $Q_{\text {eff }}^{(2)}$. The dotted line indicates the bare, unscreened charge of the polyelectrolyte, whereas the full line shows a prediction based on counterion condensation theory, with $\xi$ being the condensation parameter. 
charge, i.e. not all condensed counterions that are included in the new estimator $Q_{\text {eff }}^{(2)}$ are taken into account.

The so defined effective charge estimators $Q_{\text {eff }}^{(1)}$ and $Q_{\text {eff }}^{(2)}$ will now be used to quantify the effective friction of the polyelectrolyte-counterion compound.

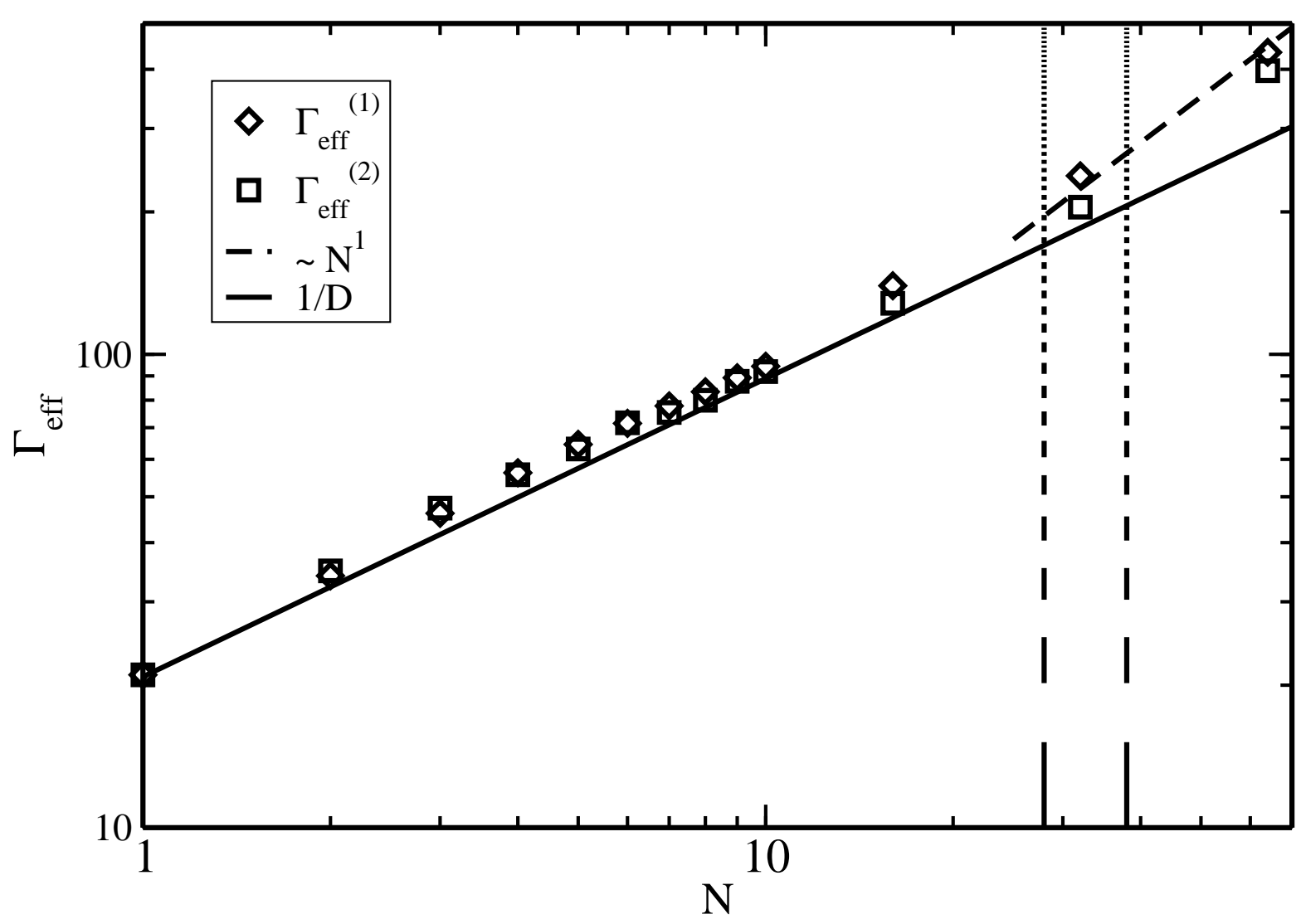

Figure 6: Effective friction of the polyelectrolyte and the co-moving counterions as abtained from (9) using $Q_{\text {eff }}^{(1)}$ and $Q_{\text {eff }}^{(2)}$. For comparison, the inverse diffusion coefficient is displayed, which is a measure for the hydrodynamic friction if the Einstein equation (12) holds. The dashed lines indicate the approximate Debye screening length for the system.

Figure 6 displays the effective friction of the compound in dependence of the length of the PE chain as obtained from (9) using the estimators $Q_{\text {eff }}^{(1)}$ and $Q_{\text {eff }}^{(2)}$. We compare the obtained result to the effective friction as it can be obtained via Einstein equation from measurements of the diffusion of the PE:

$$
\Gamma_{\mathrm{D}}=\frac{1}{D}
$$




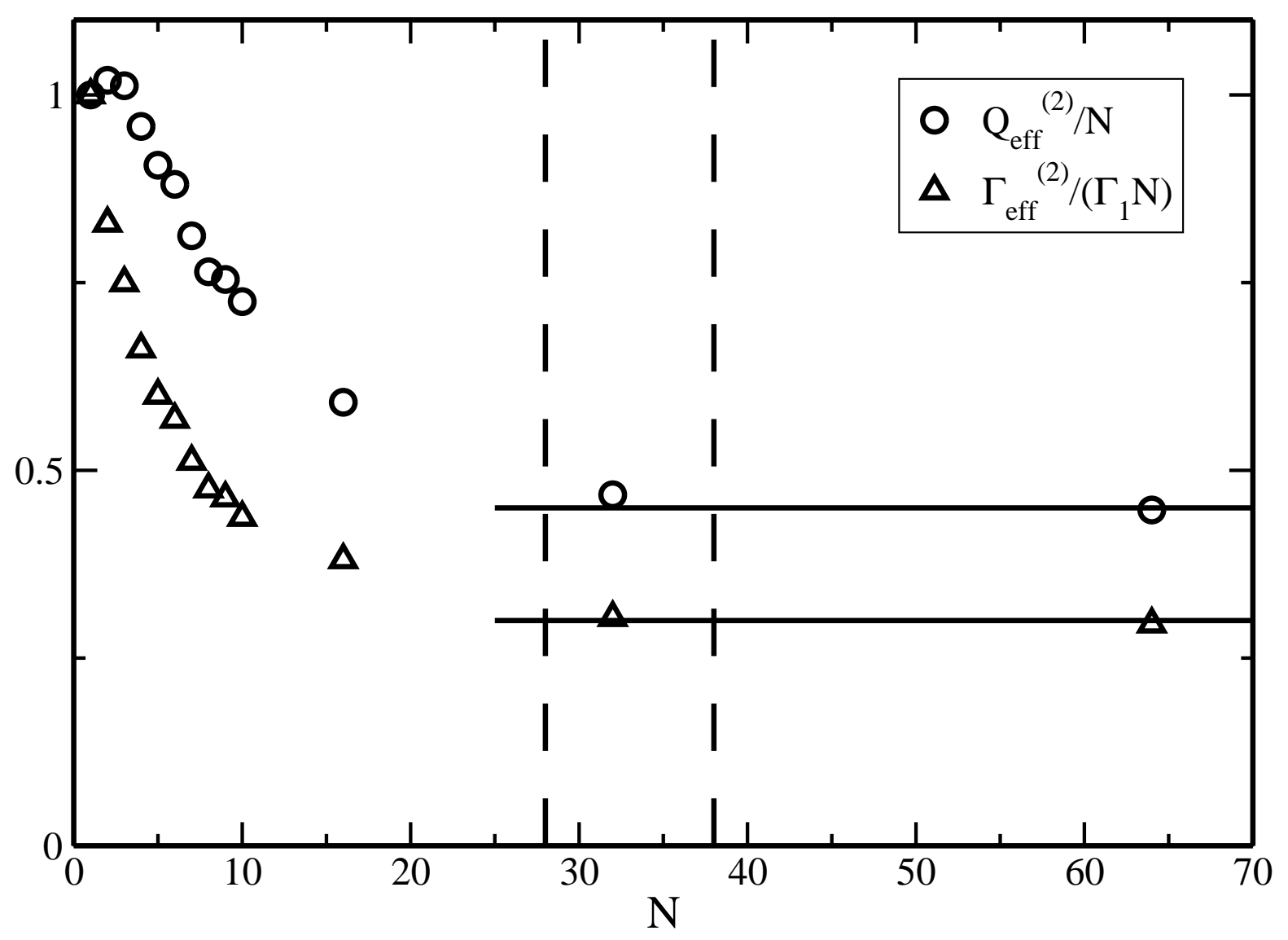

Figure 7: The effective charge and effective friction per monomer as a function of the chain length shows an initially stronger decrease of the friction compared to the charge which leads to the observed increase in mobility. For long chains, both quantities approach a constant value (the lines are visual guides only). Again the dashed lines indicate the range of electrostatic screening. 
For chains with contour lengths of the order of the Debye length, a clear deviation between $\Gamma_{\text {eff }}$ and $\Gamma_{\mathrm{D}}$ is observed. Beyond this length scale, the effective friction of the PE increases almost linearly with chain length, where as the inverse diffusion scales with an exponent of $m=0.63$ as shown in Section 3.1 .

In figure 7, we compare the effective friction and the effective charge per monomer. First, the effective friction shows a stronger decrease than the effective charge. This leads to the observed increase in mobility (see figure 4). For longer chains, both quantities approach a constant value. Again the relevant length scale seems to be the Debye length, beyond which both quantities become constant (i.e. linear scaling of $Q_{\text {eff }}$ and $\Gamma_{\text {eff }}$ ).

This gives us a microscopic understanding of the effects, that lead to the experimentally observed length independent mobility of long flexible polyelectrolytes. The hydrodynamic interactions between polyelectrolyte and counterions decrease the effective friction of the compound on length scales smaller than the electrostatic screening length. For compounds of longer chains, the hydrodynamic interactions are screened, and the friction increases linear with the size of the PE.

As an application of the presented model, we will now look at the a possible way to increase the effective friction of the compound.

\subsection{Increasing the effective friction}

As suggested by Mayer et. al. [32, 33] attaching a drag label ("molecular parachute") to one end of the PE, increases the hydrodynamic friction by a constant amount independent of the chain length. This leads to a changed scaling behaviour of the effective friction, which in turn can be seen in a restoration of the size dependent mobility even for long PE chains.

In the simulations, the label consists of $L=40$ uncharged monomers attached to one end of the PE chain. The hydrodynamic interactions with the fluid cause an additional force acting on the PE. Figure 8 demonstrates the effectiveness of this method.

In the future, we will use the toolkit described in this article to analyse the ELFSE process in more detail.

\section{Conclusions}

We used a coarse-grained MD model to describe unlabeled and labeled free-solution electrophoresis of flexible polyelectrolytes. The simulation results are in good quantitative agreement with experimental data. We used simulations without hydrodynamic interactions to efficiently measure the effective charge of the polyelectrolyte as a function of the chain length. In a further step, we quantified the effective friction of the compound formed by the poylelectrolyte and co-moving counterions. Our results show that on a length scale that is of the order of the Debye length for electrostatic screening the friction becomes linear in terms of the chain length. This increase in friction is exactly canceled out by the likewise linearly increasing effective charge, leading to the well-known constant mobility for long flexible polyelectroyte chains. 


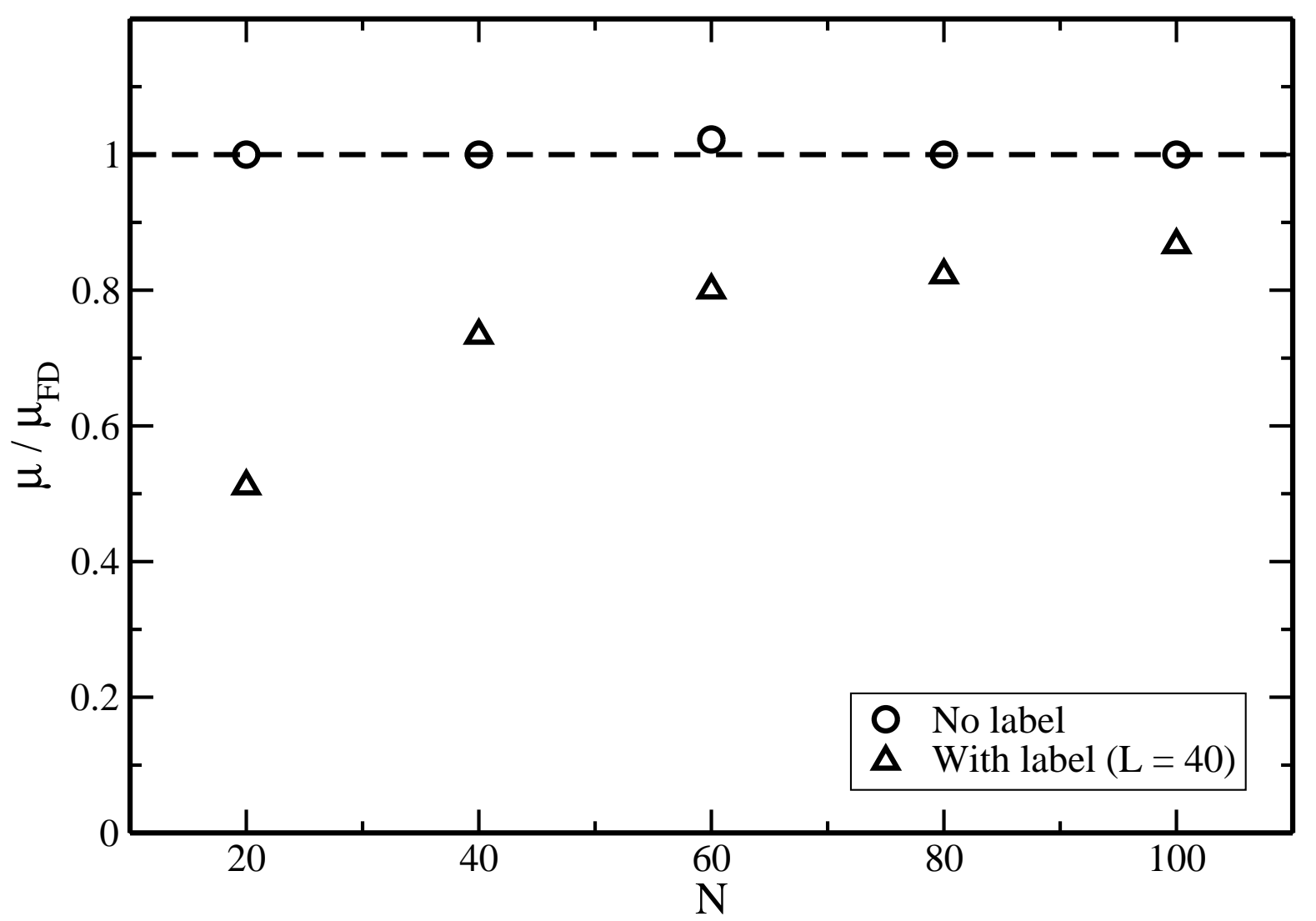

Figure 8: The mobility of a long flexible polyelectolyte without label is independent of the chain length $N$ (the line is a visual guide only). With a label of $L=40$ uncharged beads attached to the polyelectrolyte, the size dependence of the electrophoretic mobility can be recovered. 


\section{Acknowledgments}

We thank B. Dünweg, U. Schiller, and G. Slater for helpful remarks. Funds from the Volkswagen Foundation, the DAAD, and DFG under Grant No. TR6 are gratefully acknowledged.

\section{References}

[1] P. G. Righetti, editor. Capillary Electrophoresis in Analytical Biotechnology. CRC Press, Boca Raton, 1996.

[2] H. Cottet, C. Simo, W. Vayaboury, and A. Cifuentes. Nonaqueous and aqueous capillary electrophoresis of synthetic polymers. Journal of Chromatography A, 1068(1):59-73, March 2005.

[3] V. Dolnik. Capillary electrophoresis of proteins 2003-2005. Electrophoresis, 27(1):126141, January 2006.

[4] Jean-Louis Barrat and Jean-François Joanny. Theory of polyelectrolyte solutions. Advances in Chemical Physics, 94:1-66, 1996.

[5] M. Muthukumar. Theory of electrophoretic mobility of a polyelectrolyte in semidilute solutions of neutral polymers. Electrophoresis, 17(6):1167-1172, June 1996.

[6] A. R. Volkel and J. Noolandi. On the mobility of stiff polyelectrolytes. J. Chem. Phys., 102(13):5506-5511, April 1995.

[7] U. Mohanty and N. C. Stellwagen. Free solution mobility of oligomeric dna. Biopolymers, 49(3):209-214, March 1999.

[8] E. Stellwagen and N. C. Stellwagen. Determining the electrophoretic mobility and translational diffusion coefficients of dna molecules in free solution. Electrophoresis, 23(16):2794-2803, August 2002.

[9] D. A. Hoagland, E. Arvanitidou, and C. Welch. Capillary electrophoresis measurements of the free solution mobility for several model polyelectrolyte systems. Macromolecules, 32(19):6180-6190, September 1999.

[10] H. Cottet, P. Gareil, O. Theodoly, and C. E. Williams. A semi-empirical approach to the modeling of the electrophoretic mobility in free solution: Application to polystyrenesulfonates of various sulfonation rates. Electrophoresis, 21:3529-3540, 2000.

[11] E. Stellwagen, Y. J. Lu, and N. C. Stellwagen. Unified description of electrophoresis and diffusion for dna and other polyions. Biochemistry, 42(40):11745-11750, October 2003.

[12] Kai Grass, Ute Böhme, Ulrich Scheler, Hervé Cottet, and Christian Holm. Importance of hydrodynamic shielding for the dynamic behavior of short polyelectrolyte chains. Physical Review Letters, 100:096104, 2008. 
[13] H. J. Limbach, A. Arnold, B. A. Mann, and C. Holm. ESPResSo - an extensible simulation package for research on soft matter systems. Comp. Phys. Comm., 174(9):704-727, May 2006.

[14] T. Soddemann, B. Dünweg, and K. Kremer. A generic computer model for amphiphilic systems. Eur. Phys. J. E, 6:409, 2001.

[15] J. D. Weeks, D. Chandler, and H. C. Andersen. Role of repulsive forces in determining the equilibrium structure of simple liquids. J. Chem. Phys., 54:5237, 1971.

[16] M. Deserno and C. Holm. How to mesh up Ewald sums. I. A theoretical and numerical comparison of various particle mesh routines. J. Chem. Phys., 109:7678, 1998.

[17] P. Ahlrichs and B. Dünweg. Simulation of a single polymer chain in solution by combining lattice boltzmann and molecular dynamics. J. Chem. Phys., 111(17):8225-8239, 1999.

[18] V. Lobaskin, B. Dünweg, M. Medebach, T. Palberg, and C Holm. Electrophoresis of colloidal dispersions in the low-salt regime. Phys. Rev. Lett., 98:176105, April 2007.

[19] B. Dünweg, V. Lobaskin, K. Seethalakshmy-Hariharan, and C. Holm. Colloidal electrophoresis: scaling analyisis, green-kubo relation, and numerical results. Journal of Physics: Condensed Matter, page accepted, 2008.

[20] A. E. Nkodo, J. M. Garnier, B. Tinland, H. Ren, C. Desruisseaux, L. C. McCormick, G. Drouin, and G. W. Slater. Diffusion coefficient of dna molecules during free solution electrophoresis. Electrophoresis, 22(12):2424-2432, Aug 2001.

[21] E. O. Stejskal and J. E. Tanner. Spin diffusion measurements - spin echoes in presence of a time-dependent field gradient. J. Chem. Phys., 42(1):288-\&, 1965.

[22] Ulrich Scheler. Handbook of Polyelectrolytes and Their Application, Vol. 2, page 173ff. Tripaty, S. K. and Kumar, J. and Nalwa, H. S., 2002.

[23] A. Gottwald, P. Kuran, and U. Scheler. Separation of velocity distribution and diffusion using pfg nmr. Journal of Magnetic Resonance, 162(2):364-370, June 2003.

[24] P. Stilbs and I. Furo. Electrophoretic nmr. Current Opinion In Colloid \& Interface Science, 11(1):3-6, April 2006.

[25] U. Böhme and U. Scheler. Effective charge of poly(styrenesulfonate) and ionic strengthan electrophoresis nmr investigation. Colloids and Surfaces A, 222(1-3):35-40, July 2003.

[26] U. Böhme and U. Scheler. Effective charge of polyelectrolytes as a function of the dielectric constant of a solution. Journal of Colloid And Interface Science, 309(2):231235, May 2007. 
[27] Ute Böhme and Ulrich Scheler. Hydrodynamic size and electrophoretic mobility of poly(styrene sulfonate) versus molecular weight. Macromolecular Chemistry and Physics, 208(19-20):2254-2257, 2007.

[28] S. Frank and R. G. Winkler. Polyelectrolyte electrophoresis: field effects and hydrodynamic interactions. Europhys. Lett., 83:38004, 2008.

[29] G. S. Manning and J. Ray. Counterion condensation revisited. Journal of Biomolecular Structure and Dynamics, 16(2):461-476, 1998.

[30] L. Belloni, M. Drifford, and P. Turq. Counterion diffusion in polyelectrolyte solutions. Chem. Phys., 83:147, 1984.

[31] M. Deserno, C. Holm, and S. May. The fraction of condensed counterions around a charged rod: Comparison of Poisson-Boltzmann theory and computer simulations. Macromolecules, 33:199-206, 2000.

[32] P. Mayer, G. W. Slater, and G. Drouin. Theory of dna-sequencing using free-solution electrophoresis of protein-dna complexes. Anal. Chem., 66(10):1777-1780, May 1994.

[33] C. Heller, G. W. Slater, P. Mayer, N. Dovichi, D. Pinto, J. L. Viovy, and G. Drouin. Freesolution electrophoresis of dna. Journal of Chromatography A, 806(1):113-121, May 1998. 